\title{
A Pilot Study with Very Low-Intensity, Intermediate- Frequency Electric Fields in Patients with Locally Advanced and/or Metastatic Solid Tumors
}

\author{
Marc Salzberg $^{\mathrm{a}} \quad$ Eilon Kirson $^{\mathrm{b}} \quad$ Yoram Paltib, $^{\mathrm{b}} \quad$ Christoph Rochlitz $^{\mathrm{a}}$ \\ a University Hospital Basel, Switzerland \\ b Novocure Ltd., \\ c Technion, Haifa, Israel
}

Key Words

Electric fields - Tumor-treating fields (TTFields) .

Tumors · Metastasis · Pilot study

\section{Summary}

Background: The transmission of electric fields using insulated electrodes has demonstrated that very low-intensity, properly tuned, intermediate-frequency electric fields, termed tumor-treating fields (TTFields), selectively stunts tumor cell growth and is accompanied by a decrease in tumor angiogenesis. Patients and Methods: This open, prospective pilot study was designed to evaluate the safety, tolerability, and efficacy profile of TTFields treatment in patients with locally advanced and/or metastatic solid tumors using the NovoTTF$100 \mathrm{~A}^{\mathrm{TM}}$ device. All 6 patients were heavily pre-treated with several lines of therapy; no additional standard treatment option was available to them. TTFields treatment using continuous NovoTTF-100A lasted a minimum of 14 days and was very well tolerated. Results: No related serious adverse events occurred. Outcomes showed 1 partial response of a treated skin metastasis from a primary breast cancer, 3 cases where tumor growth was arrested during treatment, and 1 case of disease progression. One mesothelioma patient experienced lesion regression near TTFields with simultaneous tumor stability or progression in distal areas. Conclusion: Although the number of patients in this study is small, the lack of therapy toxicity and the efficacy observed in data gathered to date indicate the potential of TTFields as a new treatment modality for solid tumors, definitely warranting further investigation.
Schlüsselwörter

Elektrische Felder . TTFelder (tumor-treating fields) . Tumoren · Metastasen · Pilotstudie

\section{Zusammenfassung}

Hintergrund: Durch isolierte Elektroden übertragene elektrische Felder mit niedriger Intensität und sorgfältig abgestimmter mittlerer Frequenz (tumor-treating fields, TTFelder) können das Wachstum von Tumorzellen selektiv verhindern. Patienten und Methoden: Diese offene, prospektive Pilotstudie sollte die Sicherheit, Verträglichkeit und Wirksamkeit der TTFelder-Behandlung verabreicht durch das NovoTTF-100A ${ }^{\mathrm{TM}}$-Gerät bei Patienten mit lokal fortgeschrittenen und/oder metastasierten soliden Tumoren evaluieren. Alle 6 Patienten waren intensiv vorbehandelt; es stand keine Standardtherapie mehr für sie zur Verfügung. TTFelder wurden mindestens 14 Tage als Dauertherapie verabreicht. Ergebnisse: Es wurden keine behandlungsbedingten schwerwiegenden Nebenwirkungen dokumentiert. Eine partielle Remission einer Hautläsion eines Mammakarzinoms wurde beobachtet; bei 3 Patienten wurde eine Stabilisierung und bei einer Patientin ein Fortschreiten der Erkrankung unter Therapie beobachtet. Schlussfolgerung: Trotz der geringen Patientenzahl in der vorliegenden Studie sind die Absenz von Toxizität und die Hinweise auf eine gute Wirksamkeit vielversprechend und erfordern größere klinische Studien mit dieser neuen Therapieoption.

\begin{tabular}{|c|c|}
\hline CARGER & (c) 2008 S. Karger GmbH, Freiburg \\
\hline $\begin{array}{l}\text { Fax }+497614520714 \\
\text { E-mail Information@Karger.de } \\
\text { www.karger.com }\end{array}$ & $\begin{array}{l}\text { Accessible online at: } \\
\text { www.karger.com/onk }\end{array}$ \\
\hline
\end{tabular}

\section{KARGER}

E-mail Informat
www.karger.com 


\section{Introduction}

In the laboratory setting and in clinical practice, alternating electric fields show a wide range of effects on living tissues. At very low frequencies (below $1 \mathrm{kHz}$ ), alternating electric fields stimulate excitable tissues, such as nerve, muscle, and heart, through membrane depolarization [1]. The transmission of such fields by radiation is insignificant, and, therefore, they are usually applied directly by contact electrodes, though some applications have also used insulated electrodes. At very high frequencies (above many $\mathrm{MHz}$ ), a completely different biological effect is observed. Tissue heating becomes dominant primarily due to dielectric losses [2]. This phenomenon serves as the basis for some commonly used medical treatment modalities, including diathermy and radio-frequency tumor ablation, which can be applied through insulated electrodes [3].

It was recently demonstrated that very low-intensity, properly tuned, intermediate-frequency electric fields, termed tumortreating fields (TTFields), selectively stunt the growth of tumor cells [4]. This inhibitory effect was demonstrated in numerous proliferating cell types, while non-proliferating cells and tissues were unaffected. Interestingly, Nordenström's [5] 1989 observation that different cell types show specific intensity and frequency dependencies when intraneoplastic anodic and cathodic fields were used, is again confirmed with TTFields inhibition. At the cellular level, the TTFields effect was shown to be due to arrest of proliferation and selective destruction of dividing cells. The damage caused by the fields to the replicating cells was dependent on the orientation of the mitotic spindle in relation to the field vectors, indicating that this effect is non-thermal. Indeed, temperature measurements made within culture dishes during treatment and on the skin above treated tumors in vivo, showed no significant elevation in temperature compared to control cultures/mice. At the subcellular level, it was found that TTFields disrupt the normal polymerization-depolymerization process of microtubules during mitosis, similar to what has been seen in cells treated with agents that interfere directly or indirectly with microtubule polymerization (e.g. paclitaxel or docetaxel) [6-10]. Animal studies have confirmed the described inhibition of tumor growth following less than 1 week of TTFields treatment [4]. The growth inhibition was accompanied by a decrease in angiogenesis within the tumor, due to inhibition of endothelial cell proliferation, while no treatment-related side effects were observed.

Our group is the first to treat patients worldwide with this new therapeutic modality. This open, prospective pilot study was designed to evaluate the safety and tolerability profile of TTFields treatment and the tumor response in patients with locally advanced and/or metastatic solid tumors. A medical device specifically designed to apply intermittent electric fields through insulated electrodes was built into adhesive strips which were then fixed onto the patient's skin. The NovoTTF-100A ${ }^{\mathrm{TM}}$ instrument was developed by Novocure Ltd., Haifa, Israeli.

\section{Patients and Methods}

Prior to study commencement, the trial protocol was approved by the local Ethics Committee, and concurrence with the required standards of the Declaration of Helsinki was ensured. Patients with histologicallyproven, locally advanced or metastatic malignant tumors were recruited. Major selection criteria were: age $\geq 18$ years, at least 1 measurable lesion, tumor location accessible to field application through externally placed electrodes, ECOG performance $\leq 2$, no additional standard therapy available, and no concomitant anti-tumor therapy.

Six patients, with a median age of 66 years (range 24-76) and suffering from various cancers, were recruited, and provided written informed consent. All patients were previously treated with several lines of therapy, and no additional standard treatment option was available to them. Four of the patients suffered from skin lesions, 1 had a glioblastoma multiforme (GBM), and 1 had metastases from a mesothelioma in the retroperitoneal cavity (table 1). Therapy was initiated in the outpatient clinic of the Basel University Hospital under medical supervision for the first 6 hours of treatment. Thereafter, patients were released to continue treatment on an ambulatory basis. Safety and tolerability parameters were determined.

The NovoTTF-100A device used in this trial (depicted in figs. 1 and 2) is a portable, battery-operated device that produces TTFields. These TTFields are applied to the patient by means of surface electrodes that are electrically insulated, thereby ensuring that resistively coupled electric currents are not delivered to the patient. The electrodes are placed on the patient's shaved skin over a layer of adhesive hydrogel, and held in place with hypoallergenic adhesive strips. The gel beneath the electrodes must be replaced every 3-4 days, and the skin re-shaved in order to maintain optimal coupling between the electrodes and the skin. All treatment parameters are pre-set so there are no electrical output adjustments available to, or required by, the patient. Patients received continuous TTFields treatment at $100-200 \mathrm{kHz}$ at a field intensity of $0.7 \mathrm{~V} / \mathrm{cm}$ root mean square (RMS). TTFields were applied to 2 pairs of insulated electrode arrays in an alternating fashion at $1 \mathrm{~s}$ per pair. Each electrode had a surface area of $4.5-13.5 \mathrm{~cm}^{2}$. The 2 pairs were arranged normal to each other so as to gen-

Table 1. Demographics

\begin{tabular}{llll}
\hline Patient, \# & Date of initial diagnosis & Primary tumor & Location of treated lesion \\
\hline 1 & $22 / 05 / 1998$ & invasive ductal breast cancer & right chest wall, axillary skin lesions \\
2 & $08 / 11 / 2002$ & malignant melanoma & left thigh, skin lesions \\
3 & $31 / 03 / 2003$ & pleural mesothelioma & regional spread to retroperitoneum \\
4 & $23 / 10 / 2000$ & adenocarcinoma of the breast & left chest wall, skin lesion \\
5 & $05 / 09 / 2002$ & glioblastoma multiforme & left hemisphere of brain \\
6 & $18 / 02 / 2003$ & invasive ductal breast cancer & left chest wall, skin lesion \\
\hline
\end{tabular}


Fig. 1. Picture of NovoTTF portable device.

Fig. 2. Treatment setting with NovoTTF for malignant glioblastoma. Picture shows attached electrodes.
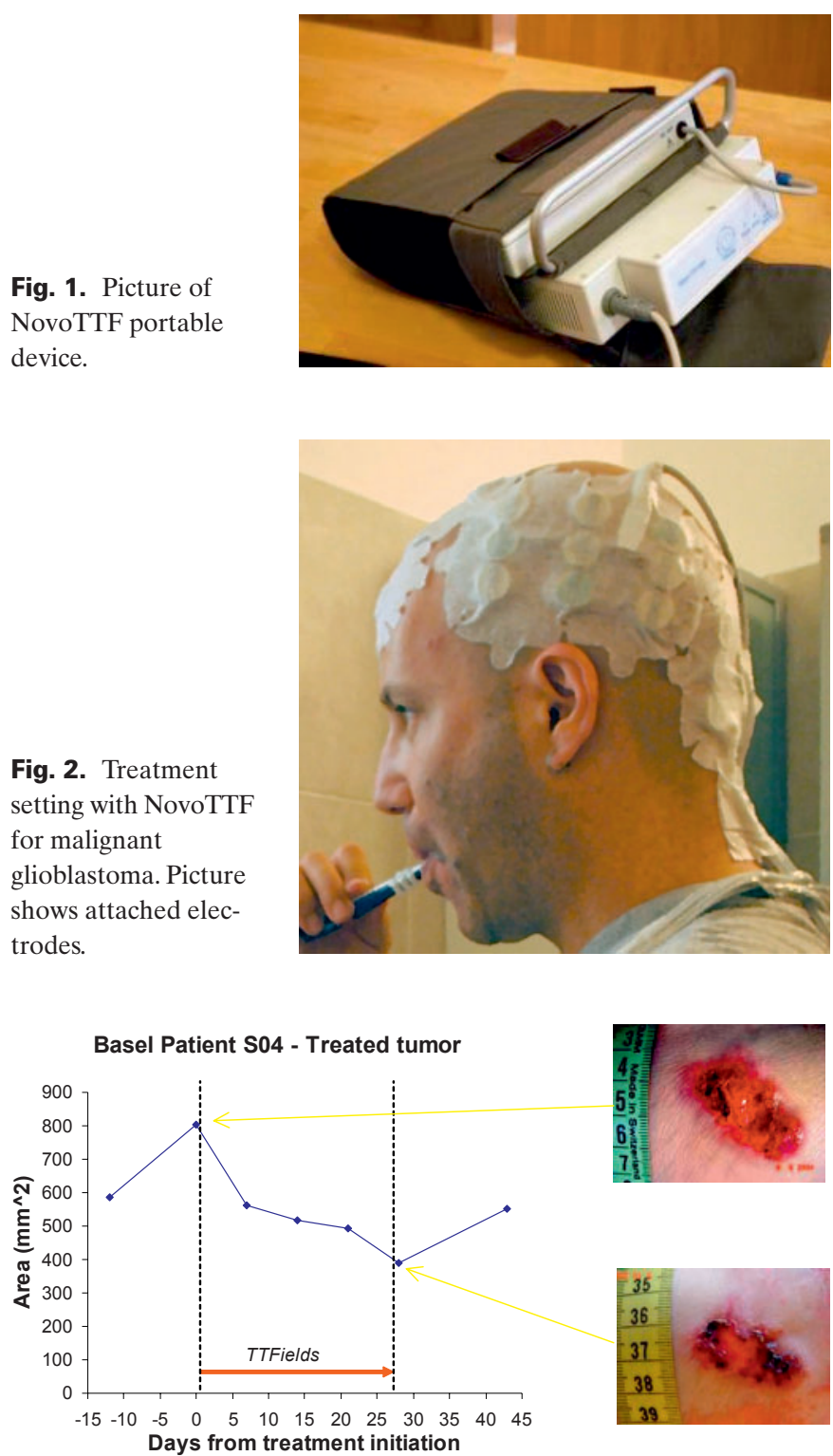

Fig. 3. Patient with a $51 \%$ reduction in tumor size after 4 weeks of TTFields treatment (partial response).

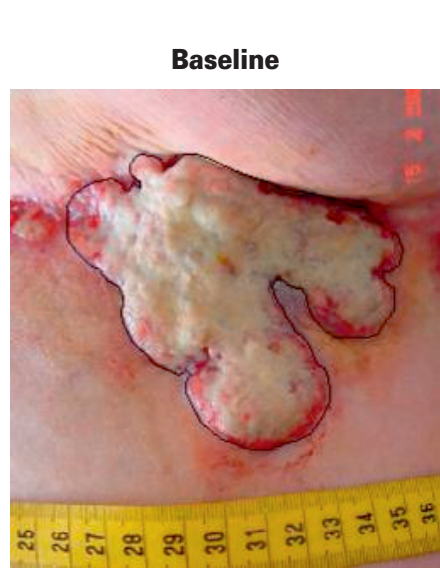

After NovoTTF treatment (4 weeks)

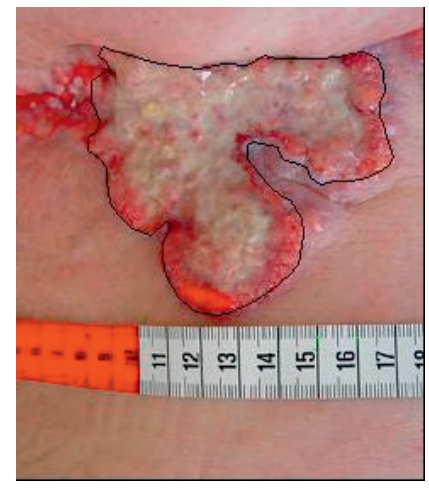

Fig. 4. Patient with flattening of the tumor after treatment and the appearance of healthy looking granulation tissue at the tumor margins. $20 \%$ reduction in tumor size after 6 weeks of TTFields treatment (stable disease). erate sequentially 2 perpendicular fields in the tumor positioned in between them $[4,11]$. The first 2 patients recruited received 2 weeks of continuous TTFields therapy. From patient 3 onwards, all patients received at least 4 weeks of continuous treatment. Patients were allowed to disconnect from the device for up to $30 \mathrm{~min}$, twice a day.

\section{Results}

Safety

The total exposure time of the 6 patients to TTFields treatment was 128 full days. Individual patients were exposed to NovoTTF-100A treatment for 13-46 days. The TTFields treatment was generally well tolerated, and the compliance was $>80 \%$. Time without treatment was due to battery changes, electrode gel replacement, and time taken by the patient for personal needs (e.g. bathing). The patients learned rapidly to manage normal daily life with the NovoTTF100A. The only improvement most patients suggested was that the device should become lighter and less noisy. Adverse events were mild for all patients. The only adverse event related to treatment was a grade 1 skin irritation, with reddening of the skin in 3 out of 6 patients. These lesions occurred beneath the electrodes, and were reversible. Treatment of the skin lesions included the repositioning of the electrodes and topical application of steroid-containing ointments. No related abnormal laboratory values or serious adverse events were recorded.

\section{Efficacy}

All patients suffered from progressive disease prior to entering the study, and all were intensively pre-treated. Tumor size was assessed by digital photography in the 4 patients with skin tumors as the measurable lesion, and the other 2 patients by computed tomography (CT) scans. One partial response of a treated skin metastasis of a primary breast cancer was observed (fig. 3 ). In 3 patients, an arrest of tumor growth during treatment was seen (fig. 4), and 1 patient experienced progressive disease. In the mesothelioma patient (Patient 3), some tumor regression was seen in the area of the tumor which was exposed to TTFields, while the other portions of the tumor were stable or progressive. Patient 5 with rapidly growing GBM resistant to temozolomide and carmustine, likewise did not respond to the 4 weeks of treatment with TTFields. On the basis of the treatment data subsequently obtained on GBM patients, we can possibly attribute this failure to the treatment duration being too short [4].

\section{Discussion}

TTFields are a new cancer treatment modality that has shown a favorable tolerability and efficacy profile in preclinical studies. We report the results of the first study with TTFields in humans, and confirm, in the clinical setting, the feasibility 
of the TTFields treatment with the NovoTTF device. Other subsequently conducted studies confirm these findings [4]. Furthermore, patients experienced very low toxicity as a consequence of this treatment, which can be explained in light of the known passive electric properties of normal tissues within the body and the effects of electric fields applied via insulated electrodes. More specifically, 2 types of toxicities may be expected in an electric field-based treatment modality. First, the fields could interfere with the normal function of excitable tissues within the body causing, in extreme cases, cardiac arrhythmias and seizures. However, this is not truly a concern with TTFields since, as frequencies increase above $1 \mathrm{kHz}$, excitation by alternating sinusoidal electric fields decreases dramatically due to the parallel resistor-capacitor nature of the cell membrane which has a time constant of about $1 \mathrm{~ms}$. Secondly, the anti-mitotic effect of TTFields might be expected to damage the replication of rapidly dividing healthy cells within the body (bone marrow, small intestine mucosa). The lack of damage to intestinal mucosa in animals undergoing TTFields treatment is probably a reflection of the fact that the small intestine mucosal cells have a slower replication cycle than neoplastic cells, and that the fraction of the field that affects the mucosal areas where the cells replicate is small due to bypassing lower resistance pathways. Bone marrow is almost completely naturally protected from TTFields due to its high electric resistance from both the surrounding bone and bone marrow itself, relative to other tissues in the body.

TTFields therapy was very well tolerated and safe. The 4 patients with skin lesions showed transient yet convincing inhibition in the growth rate of the treated lesions. One of these patients had a partial response to treatment. Although the number of patients in this study is small, the lack of toxicity of this therapy and the promise of efficacy seen in the data gathered to date indicate the potential of TTFields as a new treatment modality for solid tumors, definitely warranting further investigation in larger clinical trials.

\section{References}

1 Polk C: Therapeutic applications of low-frequency sinusoidal and pulsed and magnetic electric fields in Bronzino JD (ed): The Biomedical Engineering Handbook. Boca Raton, FL, CRC Press, Inc., 1995, pp. 1404-16.

2 Elson E: Biologic effects of radiofrequency and microwave fields: in vivo and in vitro experimental results; in Bronzino JD (ed): The Biomedical Engineering Handbook. Boca Raton, FL, CRC Press, Inc., 1995, pp. 1417-23.

3 Chou CK: Radiofrequency hyperthermia in cancer therapy; in Bronzino JD (ed): The Biomedical Engineering Handbook. Boca Raton, FL, CRC Press, Inc., 1995, pp. 1424-30.

4 Kirson E, Dbaly V, Tovarys F, Vymazal J, Soustiel J, Itzhaki A, Mordechovich D, Steinberg-Shapira S, Gurvich Z, Schneidermann R, Wassermann Y, Salzberg M, Ryffel B, Goldsher D, Dekel E, Palti Y: Alternating electric fields arrest cell proliferation in animal tumor models and human brain tumors. Proc Natl Acad Science U S A 2007;104:10152-57.
5 Nordenström BE: Electrochemical treatment of cancer. I: Variable response to anodic and cathodic fields. Am J Clin Oncol 1989;12:530-6.

6 Jordan MA, Thrower D, Wilson L: Effects of vinblastine, podophyllotoxin and nocodazole on mitotic spindles: implications for the role of microtubule dynamics in mitosis. J Cell Sci 1992;102: 401-16.

7 Rowinsky EK, Donehower RC: Paclitaxel (taxol). N Engl J Med 1995;332:1004-14.

8 Kline-Smith SL, Walczak CE: The microtubuledestabilizing kinesin XKCM1 regulates microtubule dynamic instability in cells: Mol Biol Cell 2002;13:2718-31.
9 Kapoor TM, Mayer TU, Coughlin ML, Mitchison TJ: Probing spindle assembly mechanisms with monastrol, a small molecule inhibitor of the mitotic kinesin, Eg5. J Cell Biol 2000;150:975-88.

10 Maiato H, Sampaio P, Lemos CL, Findlay J, Carmena M, Earnshaw WC, Sunkel CE: MAST/ Orbit has a role in microtubule-kinetochore attachment and is essential for chromosome alignment and maintenance of spindle bipolarity. J Cell Biol 2002;157:749-60.

11 Kirson E, Gurvich Z, Schneiderman R, Dekel E, Itzhaki A, Wasserman Y, Schatzberger R, Palti Y: Disruption of cancer cell replication by alternating electric fields. Cancer Res 2004;64:3288-95. 